\title{
A Case of Crohn's Disease with Improvement after Azathioprine-Induced Pancytopenia
}

\author{
Yong Sung Choi ${ }^{a}$ Jung Pil Suh ${ }^{a}$ Kee Ho Song ${ }^{b}$ \\ Jae Bum Lee Doo Seok Lee $^{b}$ In Taek Lee ${ }^{b}$ Do Sun Kim ${ }^{b}$ \\ Doo Han Lee ${ }^{b}$ \\ Departments of a Gastroenterology and bSurgery, Daehang Hospital, Seoul, Korea
}

\section{Key Words}

Crohn's disease $\cdot$ Improvement $\cdot$ Pancytopenia

\begin{abstract}
The immunosuppressant azathioprine (AZA) is widely used in the treatment of inflammatory bowel disease (IBD) for both inducing and maintaining remission. However, the adverse effects of AZA can often necessitate a dose reduction or discontinuation. Bone marrow suppression is one of the most serious complications with AZA treatment. On the other hand, some reports have suggested that neutropenia during AZA therapy reduced the relapse rates of IBD patients, and there have been some cases where eradication of the sensitized leukocytes by leukapheresis or bone marrow transplantation improved the IBD, which may explain the relevant role of neutropenia in controlling disease activity. This report describes the case of a 22-year-old male patient who had Crohn's colitis and complicated perianal fistulas that required immunosuppression; he achieved endoscopically determined remission and showed accelerated mucosal healing as well as clinical remission following the AZA-induced pancytopenia.
\end{abstract}

\section{Introduction}

A rising trend in the incidence and prevalence of inflammatory bowel disease (IBD) in Asia has been recognized for the past two decades. The thiopurine drugs azathioprine (AZA) and mercaptopurine are widely used for the treatment of IBD and have proven to be effective in both inducing and maintaining remission of Crohn's disease and ulcerative colitis; however adverse effects can occur in $9-28 \%$ of patients, and these adverse effects 
often necessitate a dose reduction or discontinuation. Bone marrow suppression is one of the most serious complications with AZA treatment. Nevertheless, some reports suggested that neutropenia during AZA therapy reduced the relapse rates in IBD patients $[1,2]$, and that the eradication of sensitized leukocytes, which was done by leukapheresis or bone marrow transplantation, improved IBD in some cases $[3,4]$. This may explain the relevant role of neutropenia in controlling disease activity. There have also been some cases of ulcerative colitis with prolonged remission following AZA-induced pancytopenia $[5,6]$.

\section{Case Report}

A 22-year-old man with a known history of Crohn's colitis who had undergone fistulectomy for complex perianal fistula 2 years previously was admitted with a complaint of severe weight loss $(25 \mathrm{~kg}$ during 2 years), diarrhea, abdominal pain and recurrent perianal tenderness. His home medications were mesalamine and herbal medicine. Physical exam revealed fistular openings and subcutaneous abscess pockets with severe tenderness on the anus and buttock. He was not able to maintain a sitting or supine position, so his social activity was limited. The vital signs were normal except for mild fever. Examination under anesthesia revealed complicated anal fistulas and perianal abscesses. On colonoscopy, multiple and diffuse aphthous ulcerations were noted (fig. 1). Contrast-enhanced computed tomography scan of the abdomen showed multifocal inflammatory wall thickening and thick-walled abscess pockets with the beaded appearance of subcutaneous small abscesses in the precoccygeal and perianal area (ig. 2 , fig. 3). The Crohn's disease activity index (CDAI) was 244. Abscess drainage and a seton operation were performed, then AZA was administered at a starting dose of $25 \mathrm{mg}$; this was increased up to $50 \mathrm{mg}$ after 2 weeks. He then revisited the hospital and presented with high fever $\left(39.2^{\circ} \mathrm{C}\right)$ and myalgia. On general peripheral blood test, leukocytes were $180 / \mu \mathrm{l}$ (segmented cells $6 \%$ ), hemoglobin was $6.6 \mathrm{~g} / \mathrm{dl}$ and platelets were $48,000 / \mu \mathrm{l}$. ESR was $55 \mathrm{~mm} / \mathrm{h}$ and CRP was $24.7 \mathrm{mg} / \mathrm{l}$. The remainder of the laboratory data was within normal limit. We immediately discontinued AZA and suspected pancytopenia and septicemia caused by the immunosuppressant. After 2 weeks of administering human recombinant granulocyte colony-stimulating factor and broad-spectrum antibiotic therapy, his fever subsided and the cell count fully recovered. Escherichia coli was found on blood culture, but it was sensitive to the antibiotics. Thiopurine methyltransferase (TPMT) was the wild type $\left({ }^{*} 1 /{ }^{*} 1\right)$. After recovering from cytopenia, the frequency of bowel movements decreased and the perianal pain and oozing improved gradually. Follow-up colonoscopy was performed 3 months later and demonstrated diffuse fibrotic scar instead of multiple ulcerations (ig. 4 ). He gained $10 \mathrm{~kg}$ within 6 months and is now on regular follow-up without clinical recurrence (CDAI score $=114)$.

\section{Discussion}

Bone marrow suppression is a common but potentially serious side effect of AZA treatment. The effect of AZA on bone marrow is largely dose-dependent. Therefore, a conventional immunosuppressive dose of less than $2.5 \mathrm{mg} / \mathrm{kg} /$ day causes predictable mild leukopenia. Rarely however, sudden, severe and unexpected myelosuppression, which is possibly idiosyncratic, has also been reported when low to moderate doses $(<2 \mathrm{mg} / \mathrm{kg} /$ day $)$ of the drug were used. In these cases, the cytopenic patients are vulnerable to the development of sepsis that necessitates hospital admission for intensive care.

Immunosuppression by AZA is supposed to be mediated through its effects on lymphocytes. However, the preferential suppression of neutrophils develops when leukopenia occurs, which suggests that AZA's antiinflammatory effects are mediated through its effects on neutrophils as well [7]. In fact, clinical relapse of IBD is characterized by increased neutrophil migration into the intestine [8]. Therefore, 
neutropenia following AZA can probably affect the clinical improvement, theoretically. Moreover, some reports have described cases of ulcerative colitis with prolonged remission following pancytopenia. Burke et al. reported prolonged remission in excess of 4 years following induced pancytopenia, where 3 of the 4 patients who developed severe neutropenia remained in remission for up to 21 months following that episode [6].

The mechanism that can explain this clinical improvement is unclear. First, given that altered host immunity plays an important role in IBD activity, it is possible that this patient's host immunity was reset following the pancytopenia caused by AZA. According to one report that analyzed the course of patients with Crohn's disease and ulcerative colitis and who underwent allogeneic stem cell transplantation for myeloid leukemia and myelodysplastic syndrome, 10 of 11 patients showed no IBD activity after stem cell transplantation, and the colonoscopy after complete discontinuation of prophylactic posttransplant immunosuppression revealed no pathologic findings [3]. Second, the possible benefit of granulocyte macrophage colony-stimulating factor (GM-CSF) can be hypothesized. Dieckgraefe and Korzenik investigated the safety and possible benefit of GM-CSF for the treatment of 15 patients with moderate to severe Crohn's disease. None of the patients had worsening of their disease. All 15 patients had a significant decrease in mean CDAI score during treatment [9]. Third, the original bowel flora is temporarily altered after total gut decontamination and long-term antibacterial treatment. Therefore, the most likely theory seems to be the modification of a genetically determined immune abnormality that is responsible for the chronic intestinal inflammation by the reset of the immune system or modification of an altered microbial environment. However, it is unclear whether this mechanism can affect the long-term clinical course.

Some studies have reported that TPMT genotype or activity does not predict the development of AZA-induced myelotoxicity [10-12]. On the other hand, some reports have suggested a substantial correlation between low enzyme activity and the development of myelotoxicity $[13,14]$. Colombel et al. reported that assessment of the TPMT genotype or activity can reduce the risk of myelotoxicity in approximately one-third of patients [15]. In our case, the TPMT was the wild type and this result raised doubts whether TPMT can be a reasonable screening test to identify patients with a high risk of severe myelotoxicity due to an inherited deficiency of TPMT activity.

In conclusion, we report here a patient who had Crohn's colitis and complicated perianal fistulas that required immunosuppression, and who achieved endoscopically determined remission that showed accelerated mucosal healing as well as clinical remission after AZA-induced pancytopenia. 


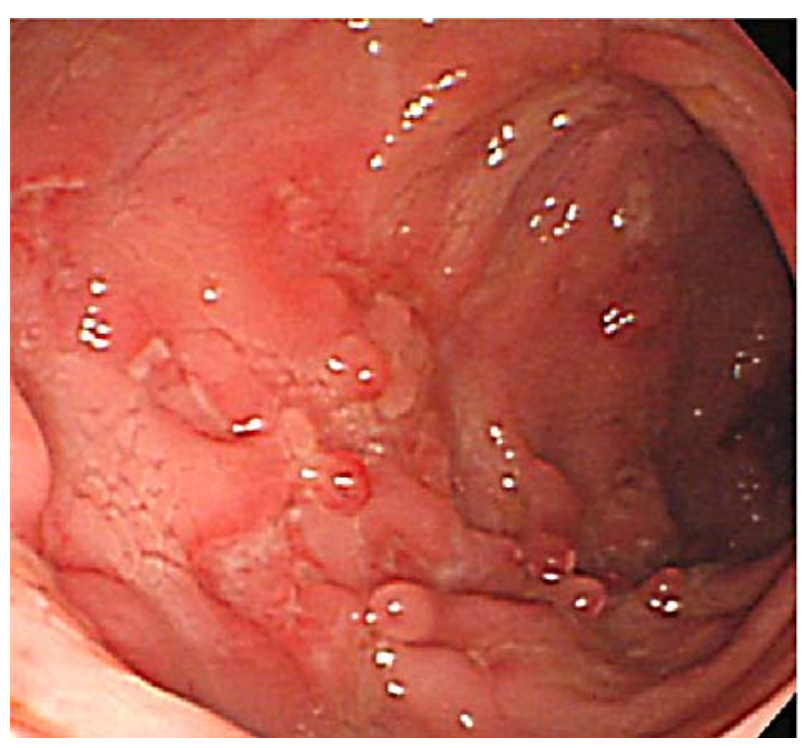

Fig. 1. Colonoscopy demonstrated multiple longitudinal aphthous ulcerations with edematous mucosal inflammation.

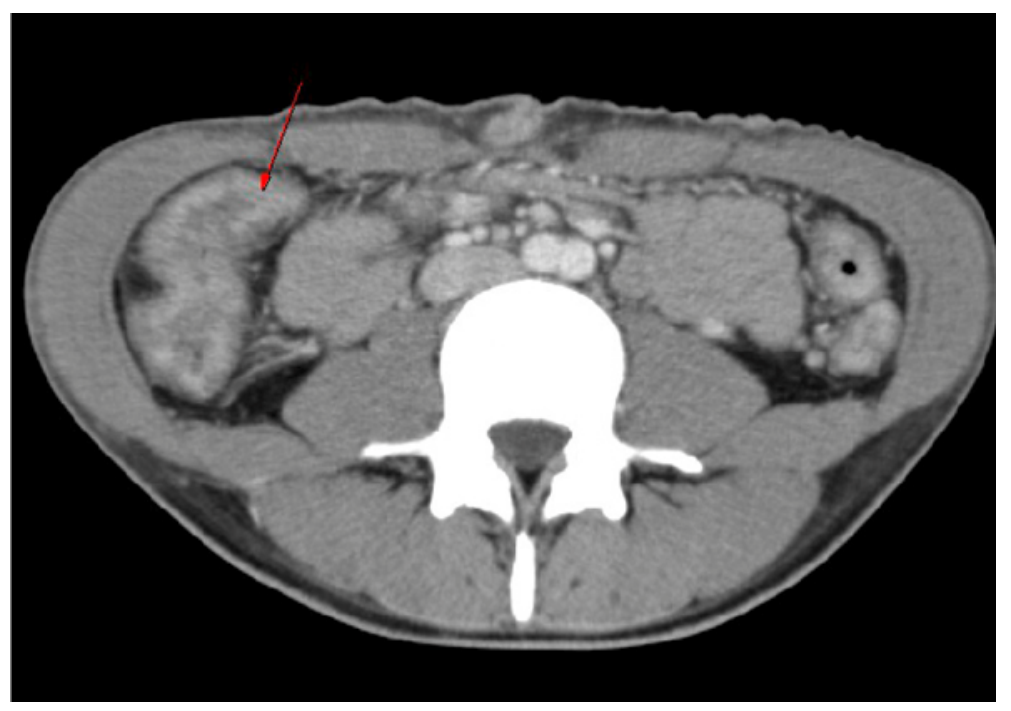

Fig. 2. Contrast-enhanced abdominal CT scan showed multifocal inflammatory colonic wall thickening on the ascending colon (arrow). 


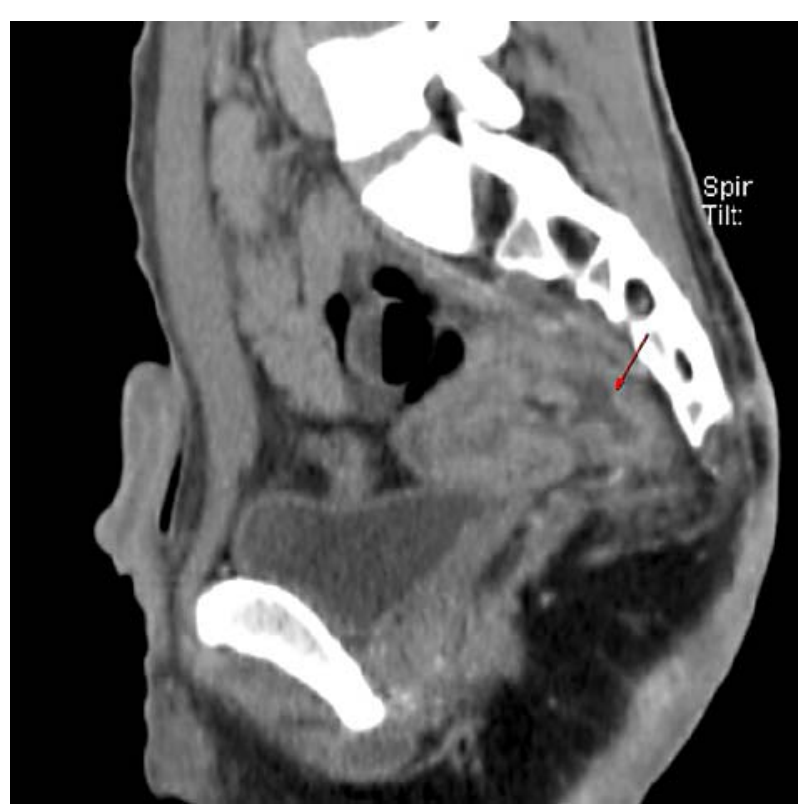

Fig. 3. Contrast-enhanced abdominal CT scan showed thick-walled abscess pockets in the precoccygeal area (arrow) and mutiple subcutaneous small abscesses with a beaded appearance.

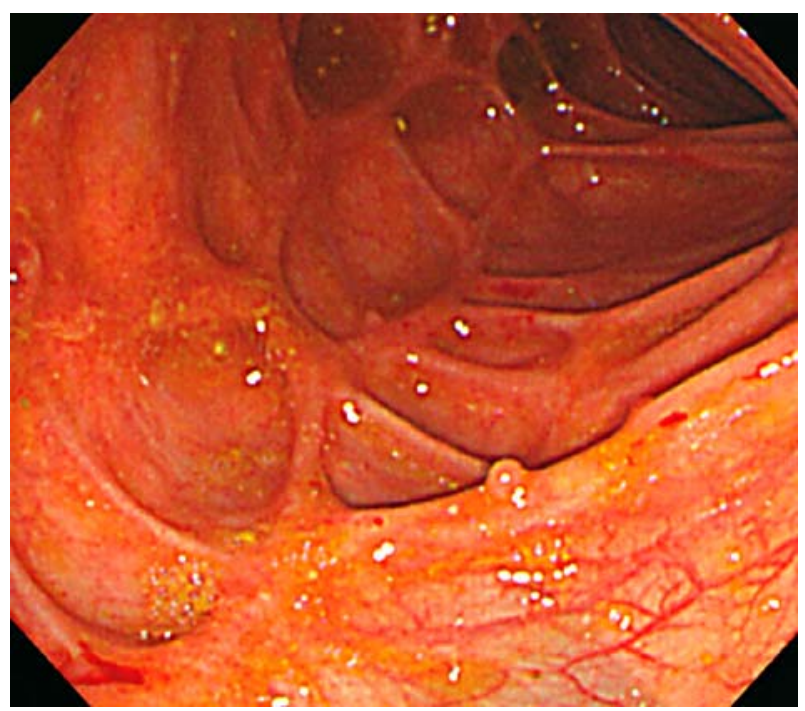

Fig. 4. Follow-up colonoscopy after recovery of pancytopenia demonstrated disappearance of the multiple active aphthous ulcerations; diffuse fibrotic scar formation (suggesting accelerated mucosal healing) was noted instead. 


\begin{tabular}{|c|c|c|c|}
\hline $\begin{array}{r}\text { Case Reports in } \\
\text { Gastroenterology }\end{array}$ & $\begin{array}{l}\text { Case Rep Gastroenterol 2011;5:344-349 } \\
\text { DOI: } 10.1159 / 000329707\end{array}$ & $\begin{array}{l}\text { Published online: } \\
\text { July 5, } 2011\end{array}$ & $\begin{array}{l}\text { @ } 2011 \text { S. Karger AG, Basel } \\
\text { ISSN 1662-0631 } \\
\text { www.karger.com/crg }\end{array}$ \\
\hline
\end{tabular}

\section{References}

$\checkmark 1$ Campbell S, Ghosh S: Is neutropenia required for effective maintenance of remission during azathioprine therapy in inflammatory bowel disease? Eur J Gastroenterol Hepatol 2001;13:1073-1076.

12 Candy S, Wright J, Gerber M, et al: A controlled double blind study of azathioprine in the management of Crohn's disease. Gut 1995;37:674-678.

-3 Ditschkowski M, Einsele H, Schwerdtfeger R, et al: Improvement of inflammatory bowel disease after allogeneic stem-cell transplantation. Transplantation 2003;75:1745-1747.

4 Suzuki Y, Yoshimura N, Saniabadi AR, Saito Y: Selective granulocyte and monocyte adsorptive apheresis as a first-line treatment for steroid naive patients with active ulcerative colitis: a prospective uncontrolled study. Dig Dis Sci 2004;49:565-571.

5 Lee JS, Park YS, Kim NI, et al: A case of ulcerative colitis with prolonged remission following azathioprineinduced pancytopenia. Intest Res 2008;6:85-89.

6 Burke DA, Dixon MF, Axon AT: Ulcerative colitis: prolonged remission following azathioprine-induced pancytopenia. J Clin Gastroenterol 1989;11:327-330.

7 Korelitz BI, Zlatanic J, Smith MJ, et al: Significance of WBC differential when leukopenia is induced by 6-MP for IBD. Gastroenterology 1997;113:1810-1811.

$\checkmark 8$ Teahon K, Bjarnason I: Comparison of leukocyte excretion and blood loss in inflammatory disease of the bowel. Gut 1993;34:1535-1538.

-9 Dieckgraefe BK, Korzenik JR: Treatment of active Crohn's disease with recombinant human granulocytemacrophage colony-stimulating factor. Lancet 2002;360:1478-1480.

10 Gearry RB, Barclay ML, Burt MJ, et al: Thiopurine S-methyltransferase (TPMT) genotype does not predict adverse drug reactions to thiopurine drugs in patients with inflammatory bowel disease. Aliment Pharmacol Ther 2003;18:395-400.

11 Reuther LO, Sonne J, Larsen NE, et al: Pharmacological monitoring of azathioprine therapy. Scand J Gastroenterol 2003;38:972-977.

12 Reuther LO, Vainer B, Sonne J, Larsen NE: Thiopurine methyltransferase (TPMT) genotype distribution in azathioprine-tolerant and -intolerant patients with various disorders. The impact of TPMT genotyping in predicting toxicity. Eur J Clin Pharmacol 2004;59:797-801.

13 Gisbert JP, Nino P, Rodrigo L, et al: Thiopurine methyltransferase (TPMT) activity and adverse effects of azathioprine in inflammatory bowel disease: long-term follow-up study of 394 patients. Am J Gastroenterol 2006;101:2769-2776.

14 Zelinkova Z, Derijks LJ, Stokkers PC, et al: Inosine triphosphate pyrophosphatase and thiopurine s-methyltransferase genotypes relationship to azathioprine-induced myelosuppression. Clin Gastroenterol Hepatol 2006;4:44-49.

15 Colombel JF, Ferrari N, Debuysere H, et al: Genotypic analysis of thiopurine S-methyltransferase in patients with Crohn's disease and severe myelosuppression during azathioprine therapy. Gastroenterology 2000;118: $1025-1030$. 